\title{
MODIFICACIONES INTENCIONALES DEL CRÁNEO. PRIMER REGISTRO DE PERFORACIONES CRANEANAS EN EL NORESTE ARGENTINO
}

\author{
INTENTIONAL MODIFICATIONS OF THE SKULL. FIRST RECORD OF CRANIAL \\ PERFORATION IN NORTHEAST ARGENTINA
}

\author{
Clara Scabuzzo y María Agustina Ramos van Raap ${ }^{2}$
}

\begin{abstract}
En las investigaciones bioarqueológicas y paleopatológicas, las modificaciones intencionales en el cráneo adquieren gran valor, dado que permiten abordar diferentes aspectos sobre los individuos y las poblaciones del pasado. Un ejemplo de modificaciones craneanas ampliamente difundido en distintas partes del mundo es la trepanación. Particularmente en Argentina, los estudios bioarqueológicos reportaron escasos ejemplos de estas intervenciones. El objetivo de este trabajo es presentar, analizar y discutir el hallazgo de perforaciones intencionales en fragmentos craneales de un infante inhumado en el sitio arqueológico Cerro Lorenzo 2 (Entre Ríos, Argentina) correspondiente al Holoceno Tardío. Se describirán las características morfológicas y métricas de las oquedades, se realizará el diagnóstico diferencial y se evaluará la técnica empleada. Este análisis se efectuó mediante la observación macroscópica y el empleo de lupas y radiografías. Los resultados indican que las oquedades se efectuaron intencionalmente en momentos perimortem y a través de la técnica de perforación. Finalmente, se discute la interpretación del caso de estudio en vinculación con las modificaciones intencionales del cráneo tomando en consideración las trepanaciones; se evalúan los posibles motivos rituales o terapéuticos relacionados con estas intervenciones y las implicancias a nivel regional de esta práctica inédita hasta el momento en el Noreste Argentino.
\end{abstract}

Palabras claves: perforaciones craneanas, diagnóstico diferencial, manipulación ritual, cuenca inferior del Río Uruguay.

In bioarchaeological and palaeopathological research, intentional modifications to the skull are of great value as they allow different aspects of past individuals and populations to be addressed. An example of cranial modifications widely reported in different parts of the world is trepanation. In Argentina, bioarchaeological studies have so far reported few examples of these interventions. The aim of this work is to present, analyze, and discuss the findings on intentional perforations in the skull of a child buried at the late Holocene archaeological site of Cerro Lorenzo 2 (Entre Ríos, Argentina). The analyzes included X-rays and a macroscopic differential diagnosis to evaluate possible etiologies. The results indicate that the cavities were made perimortem and using the drilling technique. Finally, we discuss the range of possible ritual and therapeutic reasons for this intervention and the regional implications of this unprecedented practice in Northeast Argentina.

Key words: Cranial perforation, differential diagnosis, ritual manipulation, lower basin of the Uruguay river.

En las investigaciones bioarqueológicas, el análisis del cráneo permite obtener información sobre distintos aspectos de los individuos y las poblaciones pretéritas. En términos generales, esto se debe a que, por un lado, constituye un elemento diagnóstico para reconstruir los perfiles biológicos de los individuos (Buikstra y Ubelaker 1994). Por otro lado, es un elemento considerado con alto valor simbólico por representar el lugar donde se encontraría la personalidad de los individuos (Nicklisch et al. 2018). Debido a estas particularidades es que las modificaciones en el cráneo adquieren un gran valor dentro de los estudios bioarqueológicos y paleopatológicos, ya que se pueden vincular con violencia, con intervenciones rituales o con procedimientos quirúrgicos (Nicklisch et al. 2018). Un ejemplo de modificaciones craneanas ampliamente reportado es la trepanación, que consiste en la remoción intencional de una o más porciones del neurocráneo. Esta práctica se puede realizar mediante la aplicación de distintas técnicas, tales

1 CICYTTP-CONICET, Provincia de Entre Ríos-UADER. División Arqueología, Facultad de Ciencias Naturales y Museo, Universidad Nacional de La Plata, Entre Ríos, Argentina. clarascabuzzo@hotmail.com

2 CONICET - División Arqueología, Facultad de Ciencias Naturales y Museo, Universidad Nacional de La Plata. Anexo Museo, La Plata, Buenos Aires, Argentina. ramosvanraap.ma@gmail.com 
como la perforación, el raspado y el corte (Erdal y Erdal 2011; Giuffra y Fornaciari 2017; Khudaverdyan 2016; Verano 2016a, 2016b, entre otros).

El estudio de la práctica de trepanación cobró impulso en 1867, particularmente en América, a raíz de la publicación de Paul Brocca sobre la descripción de un cráneo peruano de origen incaico (Giuffra y Fornaciari 2017; Luis y Pucciarelli 1996; Podgorny 2016; Riccomi et al. 2017; Verano 2016a). Desde ese momento hasta el presente, la cantidad de casos detectados aumentó exponencialmente y se ha reconocido el desarrollo de esta práctica en diferentes periodos temporales y en los distintos continentes (Aufderheide y Rodriguez-Martin 1998; Campillo 2011; Comşa y Sankhyan 2013; Erdal y Erdal 2011; Riccomi et al. 2017; Slepchenko et al. 2017; Verano 1997; Weber y Whal 2006). Específicamente, se ha reportado una importante cantidad de casos en Europa Central y en la región Andina (Erdal y Erdal 2011; Verano 1997). En Europa, los primeros registros de trepanaciones datan del Paleolítico superior-Mesolítico (Gresky et al. 2016; Slepchenko et al. 2017), aunque el florecimiento de esta cirugía se dio durante el Neolítico, particularmente en Francia y Gran Bretaña (Giuffra y Fornaciari 2017). En cuanto a América, las dos áreas geográficas con la mayor cantidad de casos son la Cordillera de los Andes en Perú y Bolivia y el Valle de Oaxaca en México (Kurin 2013; Verano 1997).

En Argentina, los estudios bioarqueológicos han reconocido hasta el momento escasos ejemplos de trepanaciones en grupos prehispánicos. Uno de ellos corresponde a un individuo inhumado en el sitio arqueológico Carsa (Santa Cruz, Argentina), datado en el Holoceno Tardío (Castro et al. 2009). Este hallazgo se trata de un adulto joven de sexo masculino, el cual presentaba una única oquedad tronco-cónica en el hueso frontal, de $6,1 \mathrm{~mm}$ de diámetro. Esta alteración craneana fue diagnosticada como una trepanación, y los autores propusieron que la técnica utilizada fue el barrenado mediante un instrumento lítico de punta aguda (Castro et al. 2009). Los signos macroscópicos de cicatrización del diploe y la presencia de áreas densas a nivel radiológico sugieren que el individuo sobrevivió un tiempo luego de la intervención (Castro et al. 2009). Asimismo, se registraron lesiones óseas (en cráneo y esqueleto postcraneal) atribuibles a treponematosis. Otro de los casos reportados corresponde a la localidad arqueológica Doncellas, del Noroeste Argentino. El análisis bioarqueológico de más de 140 individuos evidenció la presencia de una trepanación vinculada con tratamiento terapéutico en uno de los esqueletos correspondiente a un adulto masculino (Fuchs et al. 2017).

En este trabajo se presenta, analiza y discute el hallazgo de perforaciones intencionales en el cráneo de un individuo infantil inhumado en el sitio arqueológico Cerro Lorenzo 2 (CL-2) (departamento Gualeguaychú, Provincia de Entre Ríos, Argentina). En primer lugar, se presenta la descripción macroscópica y radiológica de las oquedades observadas. Luego, dada la ausencia de antecedentes de huesos craneanos perforados en la región, se describe el diagnóstico diferencial efectuado, en el cual se consideraron diferentes patologías óseas, traumatismos y efectos como producto de procesos tafonómicos. A continuación, se evalúa la posible técnica de manufactura empleada en la realización de las oquedades. Finalmente, se discute la interpretación del caso de estudio en vinculación con las modificaciones intencionales del cráneo tomando en consideración las trepanaciones; se evalúan los posibles motivos tanto rituales como terapéuticos relacionados con este tipo de intervenciones; y se contextualiza su hallazgo con la información bioarqueológica disponible sobre las poblaciones prehispánicas que habitaron el sur del Noreste Argentino.

\section{Modificaciones Intencionales del Cráneo. Las Trepanaciones, sus Motivos y Técnicas}

La trepanación constituye una práctica de modificación intencional del cráneo ampliamente reportada para poblaciones pretéritas de diferentes regiones geográficas. Si bien las razones por las cuales se realizaron estas intervenciones entre las distintas poblaciones y las diferentes culturas son múltiples (Khudaverdyan 2016), no es fácil establecerlas. Apoyados en las evidencias etnográficas y en los relatos de cronistas, diferentes autores han propuesto que existe una relación entre este tipo de práctica y determinados motivos de carácter terapéutico o ritual (Campillo 2011; Erdal y Erdal 2011; Riccomi et al. 2017; Verano 1997). Entre las causas terapéuticas se mencionan principalmente los traumas craneales, así como la curación de dolores de cabeza, de epilepsia y de trastornos mentales. Estos últimos han sido atribuidos a la presencia de fuerzas sobrenaturales o de espíritus malignos y demonios, los cuales podían ser expulsados a través del agujero realizado en el cráneo (Kurin 2013; Riccomi et al. 2017). Como mencionan Gresky et al. (2016), si la conexión directa entre las enfermedades que generan lesiones óseas y 
la trepanación ya es una tarea difícil en los estudios paleopatológicos, resulta un problema aún mayor vincular la presencia de trepanaciones con trastornos psíquicos u otras enfermedades "invisibles", tales como dolor de cabeza, epilepsia o migraña. En este sentido, en el registro bioarqueológico, solo es posible relacionar esta práctica con motivos terapéuticos cuando se puede observar su asociación con traumas o lesiones óseas patológicas (Giuffra y Fornaciari 2017; Gresky et al. 2016; Riccomi et al. 2017). En muchos ejemplos de trepanaciones prehistóricas donde no hay evidencias de patologías óseas y su vinculación con motivos terapeúticos es difícil de establecer, una posibilidad es asociar estas modificaciones craneanas a motivos rituales. En algunos casos específicamente, se ha planteado que este tipo de intervención ritual se practicaba en momentos postmortem y tenía como fin la manufactura de amuletos, la obtención de discos craneanos "rondelles" o la experimentación de la técnica con el fin de perfeccionarla (Giuffra y Fornaciari 2017; Khudaverdyan 2014; Kurin 2013; Slepchenko et al. 2017). En este punto es importante aclarar que mientras algunos autores incluyen a este tipo de prácticas dentro de las trepanaciones (Campillo 2011; Gresky et al. 2016), otros investigadores consideran que tales intervenciones realizadas luego de la muerte de los individuos no deben ser catalogadas como tales (Erdal y Erdal 2011; Weber y Whal 2006). En este sentido, se argumenta que el término trepanación debe circunscribirse a prácticas que se realizan en individuos vivos, ya sea con propósitos médicos, rituales o religiosos.

Al analizar el tejido óseo asociado con trepanaciones, pueden distinguirse aquellas que se presentan cicatrizadas o aquellas donde no ha ocurrido remodelación ósea. En este último caso, la ausencia de cicatrización puede responder a tres situaciones: que el individuo no haya sobrevivido a la intervención, que haya sobrevivido por un corto tiempo después de practicada la cirugía o que se haya realizado cuando este ya estaba muerto, como ocurriría mayormente en el caso de las trepanaciones rituales reportadas en distintos lugares del viejo mundo (Campillo 2011; Giuffra y Fornaciari 2017; Gresky et al. 2016). Por su parte, cuando las trepanaciones están cicatrizadas se observa remodelación ósea y formación de nuevo hueso sobre el diploe (Campillo 2011; Gresky et al. 2016; Verano 2016a). Además, mediante el uso de radiografías es posible distinguir un área circundante con actividad osteogénica (Campillo 2011; Luis y Pucciarelli 1996). En estos casos la presencia de reacción ósea indica que el individuo sobrevivió un determinado tiempo luego de la intervención (Kurin 2013). Si bien es frecuente que los individuos sometidos a la práctica de trepanación tengan una buena recuperación, en algunos ejemplos arqueológicos se ha reportado la presencia de infecciones postoperatorias que ocurrirían como resultado de estas intervenciones (Campillo 2011; Erdal y Erdal 2011; Giuffra y Fornaciari 2017; Piek et al. 1999; VelascoSuarez et al. 1992; Verano 2016a; Weber y Whal 2006). Estas infecciones pueden ser identificadas a partir de la presencia de espículas óseas o múltiples fosas vasculares en las superficies alrededor de las trepanaciones (Weber y Whal 2006).

Las tendencias reportadas en distintos grupos prehistóricos indican que este tipo de procedimientos fue practicado mayormente en individuos adultos, siendo escasos los ejemplos en subadultos (Andrushko y Verano 2008; Campillo 1984, 2011; Erdal y Erdal 2011; Gresky et al. 2016; Mariani-Costantini et al. 2000; Verano 1997). Asimismo, en distintos lugares se observa una tendencia a ser más frecuente este tipo de intervención en individuos masculinos, tal como lo reflejan los trabajos de síntesis realizados en Perú, Italia y Anatolia, Turquía (Erdal y Erdal 2011; Giuffra y Fornaciari 2017; Verano 1997). En cuanto a la localización dentro del cráneo de este tipo de modificaciones, diferentes trabajos han mostrado que los huesos mayoritariamente afectados son los parietales y el frontal (Campillo 2011; Giuffra y Fornaciari 2017; Kurin 2013).

A lo largo de la historia de la humanidad, las trepanaciones han sido realizadas a partir de diferentes procedimientos. Las principales técnicas utilizadas fueron: raspado, corte, perforado y sus distintas combinaciones (Lisowski 1967). Estos procesos fueron aplicados en diversos lugares del mundo y en diferentes momentos (Erdal y Erdal 2011). La técnica de raspado consiste en eliminar parte del tejido óseo usando un instrumento para raspar. En general, el resultado de su aplicación es una oquedad de forma ovoide con los bordes biselados y un área periférica con signos de abrasión (Weber y Whal 2006). Por su parte, la técnica de corte se caracteriza por una serie de incisiones dibujadas y rediseñadas en el cráneo, las cuales se realizan con un instrumento afilado y suelen ser de forma cuadrangular (Erdal y Erdal 2011). En cuanto a la técnica de perforación, esta implica el agujereado completo o incompleto del cráneo con el uso de un instrumento de punta. Las oquedades generadas son de forma tronco-cónicas, 
con un diámetro externo superior al interno, y cuyos orificios en general no superan los $20 \mathrm{~mm}$ de extensión (Campillo 2007, 2011; Erdal y Erdal 2011; Giuffra y Fornaciari 2017). Finalmente, en la literatura se menciona la ejecución de trepanaciones que involucran el uso combinado de las técnicas de perforación y de corte. En estos casos se hacen primero varios orificios pequeños, con la misma forma, de sección truncada y luego se los une por cortes. El área formada por los orificios es circular u ovalada (Lisowski 1967; Verano 2016b). En general, a partir de la morfología y la dimensión de las oquedades es posible inferir las técnicas aplicadas (Kurin 2013; Verano 1997). Sin embargo, en los casos donde hay cicatrización y remodelación del hueso existen más dificultades para discernir cuál fue la técnica empleada (Gresky et al. 2016; Verano 2016a, 2016b).

\section{El Caso de Estudio}

El individuo infantil (CL2-80) estudiado en este trabajo se encuentra representado únicamente por la porción derecha de la mandíbula y por 14 fragmentos de la base y la bóveda craneana. Estos fragmentos óseos presentan tamaños entre 2 y $6,5 \mathrm{~cm}$ aproximadamente y no ensamblan entre sí. En conjunto, representan menos del $50 \%$ del cráneo. De acuerdo a la erupción dental se pudo estimar que se trata de un infante de entre 12 y 18 meses de edad (Ubelaker 1987). Este individuo constituye uno de los entierros humanos hallados en el sitio Cerro Lorenzo 2 (CL-2) y forma parte de la colección bioarqueológica custodiada por el Museo de Ciencias Naturales y Arqueología Prof. Manuel Almeida (Gualeguaychú, Entre Ríos, Argentina).

Entre 1955 y 2004 el profesor Almeida realizó trabajos de campo en más de 12 sitios arqueológicos localizados en la margen derecha de la cuenca inferior del Río Uruguay (Entre Ríos, Argentina). En varios de estos sitios $(n=10)$, además de recuperar materiales arqueológicos de distinta ergología (cerámica, restos faunísticos, instrumentos líticos y óseos), se hallaron numerosos entierros humanos (Ramos van Raap y Scabuzzo 2018) que hoy conforman la colección custodiada por el museo. Como parte de sus estudios, Almeida realizó un Catálogo de Restos Humanos. En este documento, para la mayoría de los entierros, se detalla información sobre el sitio de procedencia, el sector donde fue hallado y una breve caracterización del mismo. En los últimos años los materiales depositados en esta institución vienen siendo objeto de análisis arqueológicos (Castro 2017) y bioarqueológicos (Castro y Del Papa 2015; Ramos van Raap 2018a; Ramos van Raap y Scabuzzo 2018). Los estudios bioarqueológicos se centraron en establecer el número mínimo de individuos (NMI) enterrados en cada uno de los sitios, reconstruir los perfiles biológicos, evaluar el estado de salud, profundizar el análisis de las prácticas de inhumación, e inferir la dieta a partir de análisis químicos y estudios arqueobotánicos sobre los microrrestos atrapados en el cálculo dental (Castro y Del Papa 2015; Costa Angrizani y Scabuzzo 2019; Ramos van Raap 2018a, 2020; Ramos van Raap y Scabuzzo 2018; Scabuzzo y Ramos van Raap 2019).

Uno de los sitios arqueológicos trabajados por Almeida fue Cerro Lorenzo 2 (Gualeguaychú, Entre Ríos, Argentina; Figura 1). Se trata de un montículo artificial en el cual se recuperaron abundantes vestigios arqueológicos, incluyendo entierros humanos. Para este sitio se cuenta con un único fechado radiocarbónico realizado sobre hueso humano de uno de los entierros $\left(n^{\circ} 72\right)$, que dio una cronología de $2050 \pm 60$ años AP, lo que la convierte hasta el momento en la datación más antigua para la margen derecha del Río Uruguay (Castro y Del Papa 2015). La ocupación de este sitio fue vinculada a la entidad arqueológica Goya-Malabrigo (Castro 2017). Esta correspondería al correlato arqueológico de los grupos indígenas del siglo XVI conocidos genéricamente como chaná-timbú, entre cuyos principales rasgos pueden mencionarse: la construcción de montículos de tierra o cerritos; una economía mixta de caza, pesca y recolección con una horticultura a pequeña escala de la tríada maíz, poroto y zapallo; un estilo cerámico distintivo (p.ej., apéndices zoomorfos, siluetas recortadas sobre los bordes, "campanas" de alfarería), y diversas prácticas mortuorias (entierros primarios, paquetes funerarios, acumulaciones óseas, huesos aislados). Su distribución geográfica y temporal incluye las islas y la planicie de inundación de los tramos medio e inferior del Río Paraná y el tramo inferior del Río Uruguay desde ca. 2000 años AP hasta los momentos iniciales de la llegada europea a estas regiones (Bonomo et al. 2019; Politis y Bonomo 2012, 2018).

Los análisis bioarqueológicos efectuados recientemente en la serie esqueletal del sitio permitieron estimar un número mínimo de 25 individuos, de distintas edades y de ambos sexos (Ramos van Raap y Scabuzzo 2018). Los individuos se encuentran diferencialmente representados, desde esqueletos casi completos hasta escasos elementos óseos. A partir del registro escrito del museo elaborado por 


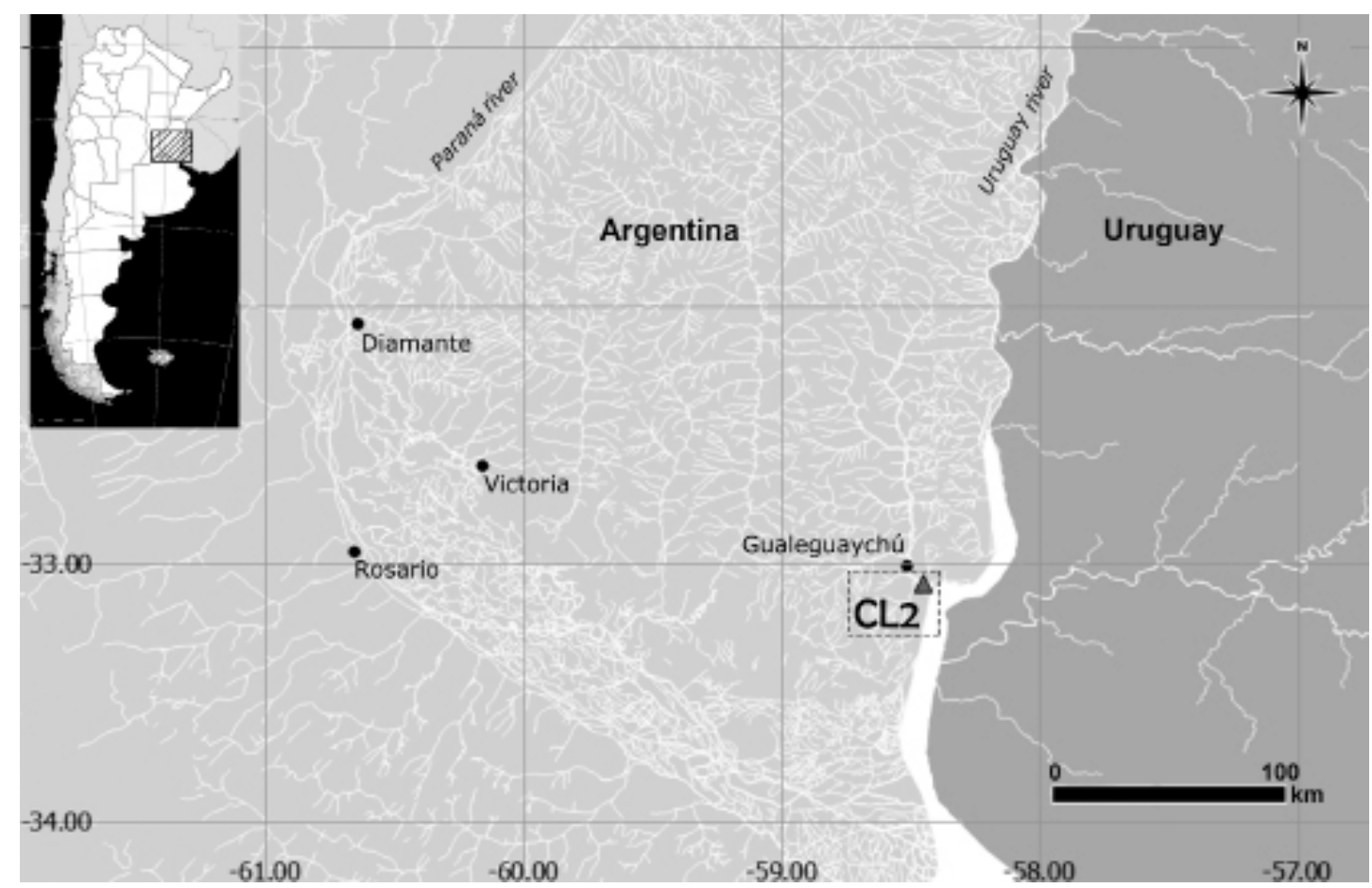

Figura 1. Mapa con la ubicación del sitio arqueológico Cerro Lorenzo 2.

Location of the Cerro Lorenzo 2 site.

el profesor Almeida (Catálogo de Restos Humanos), fue posible conocer algunas de las modalidades de entierro presentes en el sitio. Según esta información, se distinguieron inhumaciones primarias en distintas posiciones y paquetes funerarios tanto simples como múltiples (Ramos van Raap y Scabuzzo 2018). Específicamente, respecto al individuo estudiado en este trabajo, en el catálogo se consigna que los restos de este infante (CL2-80) se hallaban junto al cráneo de un individuo adulto: "Infante asociado con los individuos 78 y 79 - ubicado junto al cráneo del $\mathrm{N}^{\circ} 78$, lado derecho. Muy fragmentado. Algunos fragmentos del cráneo con extrañas perforaciones..." (Catálogo de Restos Humanos, Museo de Ciencias Naturales y Arqueología Prof. Manuel Almeida, pág s/n).

\section{Metodología de Análisis}

El análisis de los restos craneales se realizó en primer lugar de manera macroscópica y con una lupa binocular de bajo aumento (6x). Para establecer la posición de las oquedades, primero se determinó el hueso sobre el cual se encontraban las modificaciones. Debido a la fragmentación del cráneo, para poder establecer la ubicación precisa de las perforaciones sobre la bóveda, se tuvieron en cuenta rasgos anatómicos de los fragmentos (p.ej., suturas y marcas de las venas diploicas). Para esto se utilizó material comparativo de referencia y se consultaron distintos manuales de osteología. En cada perforación se midieron los diámetros máximos en $\mathrm{mm}$ y en sentido perpendicular (Buikstra y Ubelaker 1994), tanto sobre el lado externo como interno del diploe; se utilizó un calibre digital Isard con Resolución de $0,01 \mathrm{~mm}$ y Precisión de $\pm 0,02 \mathrm{~mm}$. A partir de estas medidas, y de la relación que guardan los diámetros entre sí, se determinó la morfología de cada una de las oquedades siguiendo las categorías definidas por Gresky et al. (2016): circular, subcircular, oval y ovoide. Para caracterizar la forma de la oquedad en sección, se decidió utilizar su analogía con tres cuerpos tridimensionales: cilíndrica, cónica y tronco-cónica.

En segundo lugar, con el fin de evaluar el grado de cicatrización de cada una de las oquedades, se consideraron las tres categorías propuestas por Gresky y colaboradores (2016) según las características del tejido del diploe: (1) sin cicatrización: se observa claramente la porosidad característica del diploe; (2) parcialmente cicatrizada: la porosidad del diploe es parcialmente visible; (3) completamente cicatrizada: las celdas del diploe se cubren con una capa de hueso compacto y hay márgenes suaves y redondos en la 
lesión. Simultáneamente, para evaluar de manera más precisa la cicatrización de las oquedades, se efectuaron radiografías con el fin de observar la presencia o ausencia de actividad osteogénica interna.

Una vez realizada la descripción de las oquedades según los aspectos mencionados anteriormente, se llevó a cabo el diagnóstico diferencial. Mediante este ejercicio, se evalúan las posibles causas y cuál de ellas es la más probable para explicar las condiciones óseas observadas en el caso de estudio (Ortner 2003). La aplicación del diagnóstico diferencial en este trabajo constituye una herramienta fundamental, ya que no siempre las oquedades registradas en las bóvedas craneanas son producidas de manera intencional (Khudaverdyan 2016; Verano 2016a, 2016b) y es necesario poder distinguirlas de los defectos en el cráneo que resultan de trastornos del desarrollo, de enfermedades, de traumas y de diferentes procesos tafonómicos (Aufderheide y Rodriguez-Martin 1998; Giuffra y Fornaciari 2017; Gresky et al. 2016; Ortner 2003; Verano 2016a; Weber y Whal 2006). Asimismo, en su posible vinculación con la práctica de trepanación, resulta crucial efectuar el diagnóstico diferencial cuando este tipo de casos procede de regiones geográficas donde no hay antecedentes de trepanaciones y cuando se trata de ejemplos aislados como el presentado en este trabajo (Verano 2016a, 2016b).

Finalmente, una vez realizado el diagnóstico diferencial y determinado el origen antrópico de las perforaciones, a partir de la inspección de ciertas características de las oquedades (tamaño, forma, atributos del área circundante, etc.), se evaluó la técnica que habría sido utilizada para producirlas. Asimismo, se llevó a cabo la inspección macroscópica con el fin de registrar si el individuo presentaba algún tipo de lesión ósea patológica.

\section{Resultados}

\section{Descripción de las oquedades registradas}

Del total de fragmentos del cráneo analizados, solo cuatro presentan oquedades. Tres de ellos poseen una única oquedad completa (Figura 2). El fragmento restante tiene un orificio completo y uno roto por estar sobre uno de los bordes del especímen óseo. Estos cuatro fragmentos corresponden al parietal derecho y no remontan entre sí (Figura 2). Lamentablemente, debido a que el cráneo se encuentra muy fragmentado

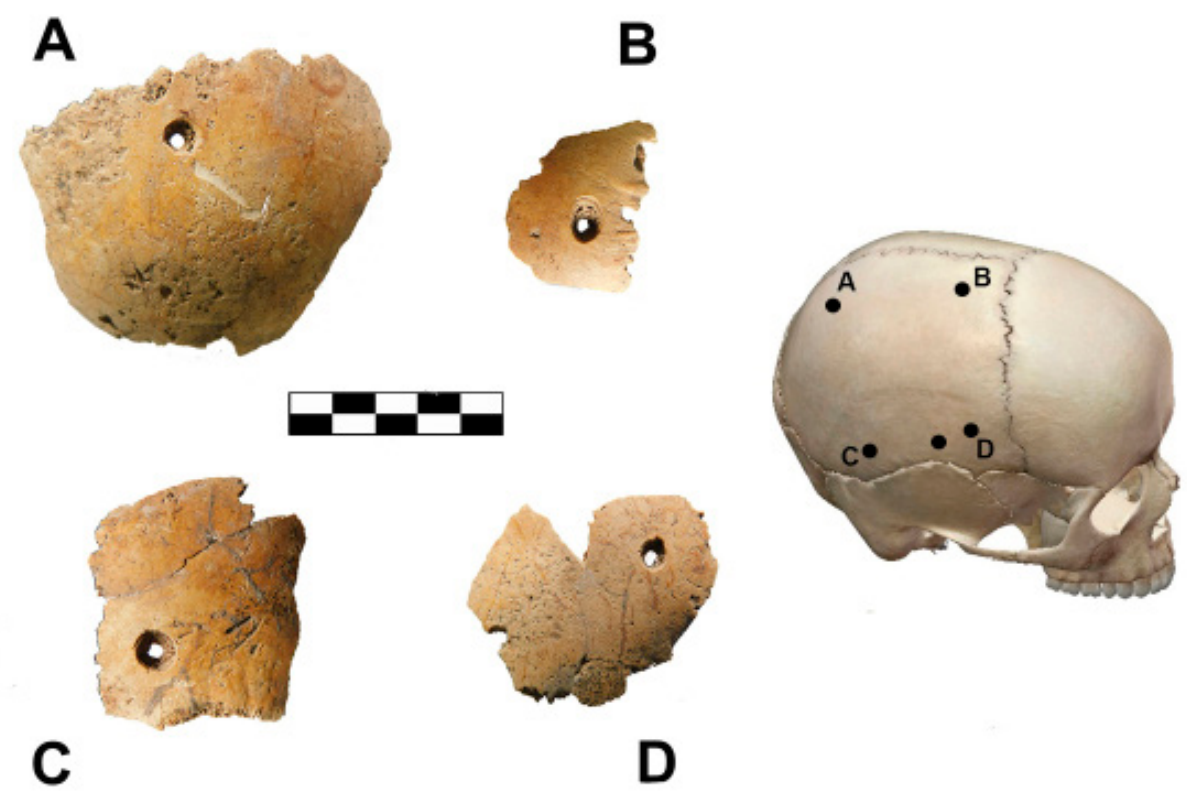

Figura 2. Conjunto de los fragmentos parietales de CL2-80 con oquedades y su posible ubicación en la bóveda craneana. A. Fragmento A con oquedad 1, B. Fragmento B con oquedad 2, C. Fragmento C con oquedad 3, D. Fragmento D con oquedades 4 y 5 .

Cranial fragments with holes and their possible location in the cranial vault. A. Fragment 1 with hole 1, B. Fragment B with hole 2, C. Fragment $C$ with hole 3, D. Fragment D with holes 4 and 5. 
e incompleto, no fue posible identificar la distancia entre las oquedades y las suturas más cercanas. El análisis macroscópico de las cinco oquedades permitió determinar que las mismas se realizaron cuando el hueso aún estaba en estado fresco. Esto último se evidencia por la ausencia de grietas o microfracturas alrededor de las perforaciones y por estar bien definidos los bordes de todas las oquedades.

Se pudieron realizar las mediciones en todas las oquedades, tanto en la cara externa como interna del diploe, a excepción de la perforación que está rota (fragmento D, oquedad 5, Tabla 1, Figura 2). Los diámetros externos muestran un rango entre 5 y $8 \mathrm{~mm}$, mientras que los diámetros internos son menores, entre 2 y $3 \mathrm{~mm}$ (Tabla 1). A partir de estas mediciones, y sobre todo considerando que los diámetros externos

Tabla 1. Medidas de los diámetros internos y externos de las oquedades registradas en el cráneo del individuo CL2-80. Measurements of the internal and external diameters of the cavities recorded in the skull of individual CL2-80.

\begin{tabular}{cccccc}
\hline Fragmento & Oquedad & $\begin{array}{c}\text { Diámetro } \\
\text { externo 1 } \\
(\mathrm{mm})\end{array}$ & $\begin{array}{c}\text { Diámetro } \\
\text { externo 2 } \\
(\mathrm{mm})\end{array}$ & $\begin{array}{c}\text { Diámetro } \\
\text { interno 1 } \\
(\mathrm{mm})\end{array}$ & $\begin{array}{c}\text { Diámetro } \\
\text { interno 2 } \\
(\mathrm{mm})\end{array}$ \\
\hline A & 1 & 5 & 5 & 2 & 2 \\
\hline B & 2 & 7 & 6 & 3 & 3 \\
\hline C & 3 & 8 & 8 & 3 & 3 \\
\hline D & 4 & 5 & 5 & 2 & 2 \\
\hline D & 5 & - & - & - & - \\
\hline
\end{tabular}

son mayores a los internos, se clasificaron todas las oquedades como de sección tronco-cónica. Por otro lado, en cuanto a la forma de las perforaciones, todas se identifican como circulares.

En cuanto al grado de cicatrización, como puede observarse en la Figura 3, no se registró remodelación ósea en ninguna de las oquedades y es posible observar claramente las celdas del diploe abiertas en cada una de ellas. Como se comentó previamente, de manera simultánea se efectuaron estudios radiográficos para evaluar la presencia de signos de actividad osteogénica en adyacencia a las oquedades. En las radiografías realizadas en los cuatro fragmentos óseos, no se observaron cambios en la coloración alrededor de las oquedades que indiquen algún tipo de actividad osteogénica en el interior del hueso producto de la cicatrización (Figura 4). Esto coincide con las observaciones macroscópicas realizadas en los bordes de los orificios.

\section{Diagnóstico diferencial}

En el diagnóstico diferencial se evaluaron los defectos en el cráneo que resultan de trastornos del desarrollo, de enfermedades, de traumas y de diferentes procesos tafonómicos. En cuanto a los defectos del desarrollo, se analizaron los meningoceles y las fenestras parietales. Los primeros consisten en depresiones circulares poco profundas que se forman
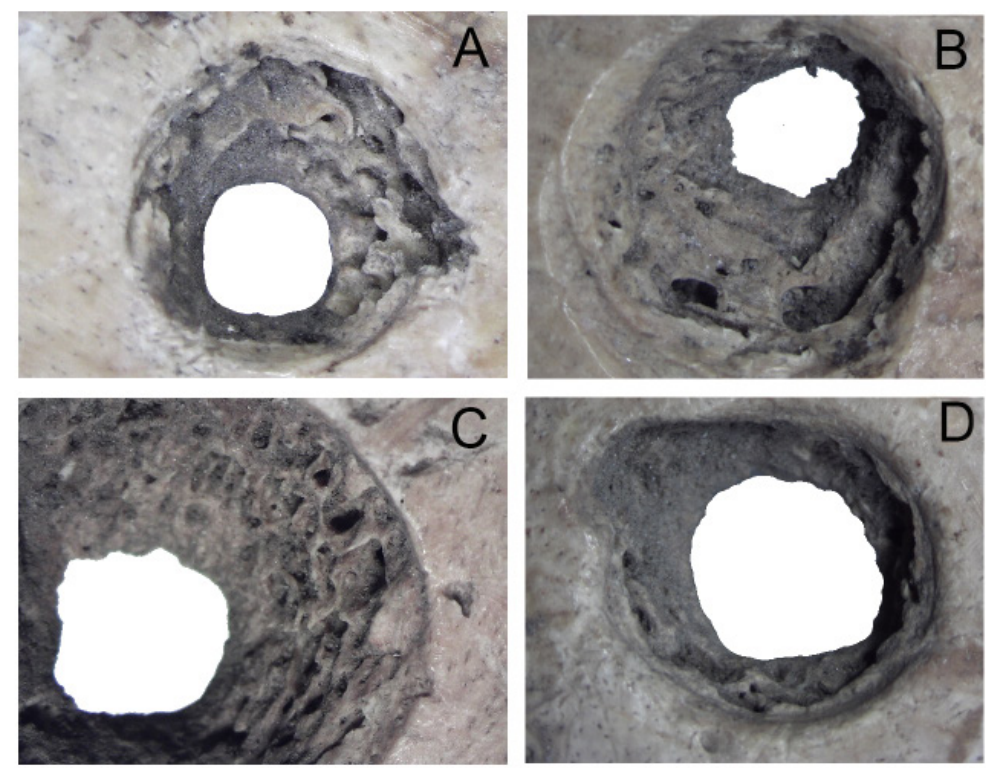

Figura 3. Detalle de las oquedades con 6x de aumento. A. Fragmento A, B. Fragmento B, C. Fragmento C, D. Fragmento D, oquedad 4.

Close-ups of holes (6x). A. Fragment A, B. Fragment B, C. Fragment C, D. Fragment D, hole 4. 


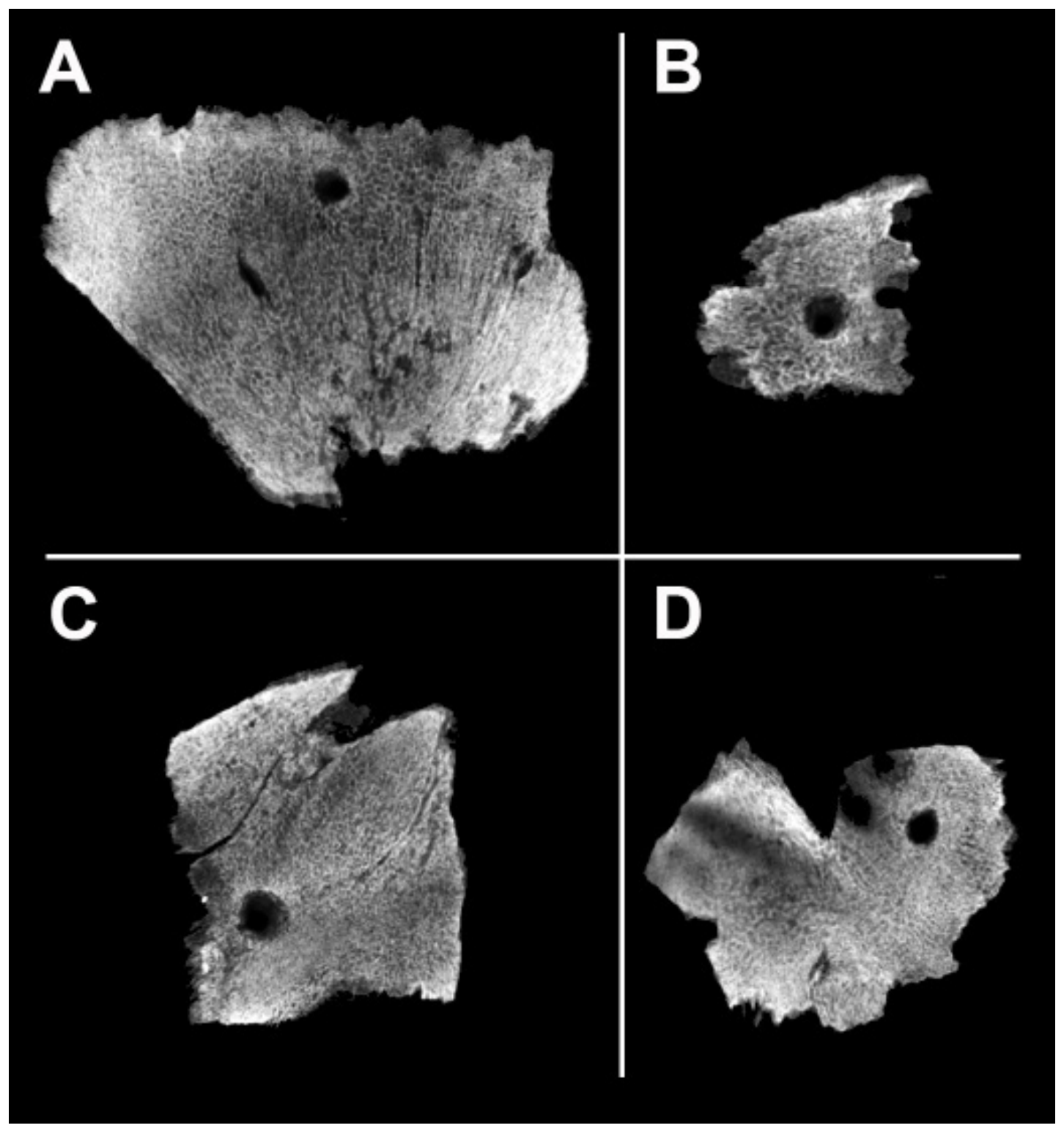

Figura 4. Imágenes radiográficas de los cuatro fragmentos craneales con las oquedades. A. Fragmento A, B. Fragmento B, C. Fragmento C, D. Fragmento D.

$X$-ray images of the four cranial fragments with cavities. A. Fragment A, B. Fragment B, C. Fragment C, D. Fragment D.

entre las suturas craneales, generalmente a lo largo de la sutura sagital. Las causas de formación de los meningoceles son fallas en la osificación durante el crecimiento y el desarrollo (Verano 2016a, 2016b). La ubicación de estos defectos en las suturas, sumado a que son depresiones y no oquedades, permiten descartar los meningoceles como causantes de los orificios observados en el individuo CL2-80. Otro tipo de defecto del desarrollo son las fenestras parietales, oquedades ovales o hendiduras que aparecen de manera bilateral en los parietales y cuyas causas de formación son desconocidas (Verano 2016a, 2016b). Tanto la forma como la simetría bilateral de estas fenestras no coinciden con las oquedades registradas en el individuo analizado y, por lo tanto, se descarta este diagnóstico.

Entre los factores patológicos que deben ser evaluados, y que se mencionan frecuentemente en la literatura especializada (Verano 2016a, 2016b), se encuentran algunas neoplasias como el mieloma 
múltiple y el carcinoma (Aufderheide y RodriguezMartin 1998). Ambos tipos de patologías se caracterizan por dejar lesiones óseas líticas con márgenes irregulares y con poca o ninguna formación ósea nueva (Ortner 2003). En el caso aquí presentado, las márgenes de las oquedades no son irregulares y su forma tronco-cónica no es compatible con las lesiones características de estas patologías neoplásicas.

Asimismo, como parte del diagnóstico diferencial también se tuvieron en cuenta distintos tipos de traumatismos. Por un lado, se evaluaron las fracturas conminutas, en las cuales el hueso se quiebra en varios fragmentos generando una oquedad irregular (Verano 2016a). La forma irregular de la oquedad no coincide con lo observado en el cráneo analizado y, además, este tipo de traumatismo no produce lesiones con forma tronco-cónica. Por lo tanto, se descarta este tipo de traumatismo como causa de las oquedades observadas en el cráneo de CL2-80. Por otro lado, las heridas penetrantes también son factibles de confundirse con trepanaciones. Estos tipos de trauma generalmente perforan el diploe y pueden adquirir diferente forma según el arma utilizada. Sin embargo, la morfología tronco-cónica característica del caso aquí expuesto no es compatible con ninguna clase de arma.

Finalmente, el daño tafonómico también debe ser considerado, sobre todo cuando no hay reacción ósea en las lesiones. Uno de los daños tafonómicos susceptible de ser confundido con una trepanación es el roído. Los roedores suelen perforar los huesos y dejar marcas características a lo largo de los márgenes de los agujeros generados, como son los surcos perpendiculares (White y Folkens 2005). Estas marcas de roído no se registraron en los fragmentos del cráneo analizado, esto lleva a que no se considere a los roedores como los causantes de las perforaciones observadas. Por otra parte, también se contemplaron las oquedades producidas por insectos. Uno de los rastros de insectos más frecuentes en los huesos son los dejados por las larvas maduras de escarabajos derméstidos (Huchet et al. 2013). Estos se caracterizan por hacer varios canales y perforaciones como resultado de la alimentación o por la excavación de las cámaras de pupación. Sin embargo, el diámetro de las perforaciones producidas por las larvas de estos insectos es en promedio ca. $3 \mathrm{~mm}$, con un rango de variación entre 0,16 y 0,64 $\mathrm{mm}$ (Huchet et al. 2013:Tabla 2). Teniendo en cuenta que los diámetros externos de las perforaciones que registramos en el cráneo son mayores (entre 5-8 $\mathrm{mm}$ ) y que la forma de las oquedades realizadas por los derméstidos no muestran la forma cono-truncada, se descarta a estos agentes como posibles causantes de las perforaciones observadas en el cráneo.

En suma, el diagnóstico diferencial realizado no permitió que se vincularan las oquedades observadas en el cráneo del infante con ninguna patología, traumatismo o proceso tafonómico. En este sentido, es posible interpretar los orificios registrados en el individuo infantil del sitio Cerro Lorenzo 2 como producto de la manipulación antrópica intencional, específicamente como resultado del agujereado intencional y reiterado del hueso parietal.

\section{Evaluación de la técnica empleada}

En cuanto al análisis de la técnica empleada para efectuar las oquedades observadas en el caso de estudio, debe tenerse en cuenta que la ausencia de cicatrización y de regeneración ósea facilita la evaluación y el reconocimiento de las acciones llevadas a cabo (Verano 2016b). Tal como se mencionó anteriormente, la técnica del raspado genera oquedades ovoides con bordes biselados y un área periférica con signos de abrasión (Weber y Whal 2006); la técnica de corte se caracteriza por una serie de incisiones que suelen ser cuadrangulares (Erdal y Erdal 2011); y la técnica de perforación produce oquedades pequeñas de formas circulares y tronco-cónicas con un diámetro externo superior al interno (Campillo 2007, 2011; Erdal y Erdal 2011; Giuffra y Fornaciari 2017). Si se tienen en cuenta las características morfológicas y tamaño de las oquedades aquí estudiadas (forma circular, sección tronco-cónica y diámetros pequeños), estas se corresponden con la ejecución de la técnica de perforación. Por otro lado, la forma en embudo de las perforaciones y el borde escalonado registrado en el fragmento B (Figura 5) indicarían que la perforación se realizó de forma manual y con un movimiento giratorio (Valenzuela Jiménez y Santos Ramírez 2013).

\section{Discusión}

La evaluación realizada sobre las oquedades en el parietal derecho de la bóveda del cráneo de un infante inhumado en el sitio Cerro Lorenzo 2 permitió constatar el primer caso de perforaciones craneanas de origen antrópico en el Noreste Argentino. Para ello fue fundamental efectuar el diagnóstico diferencial, que permitió descartar la posibilidad de que otros factores patológicos, traumáticos o postdepositacionales pudieran haber provocado tales orificios. Asimismo, la 


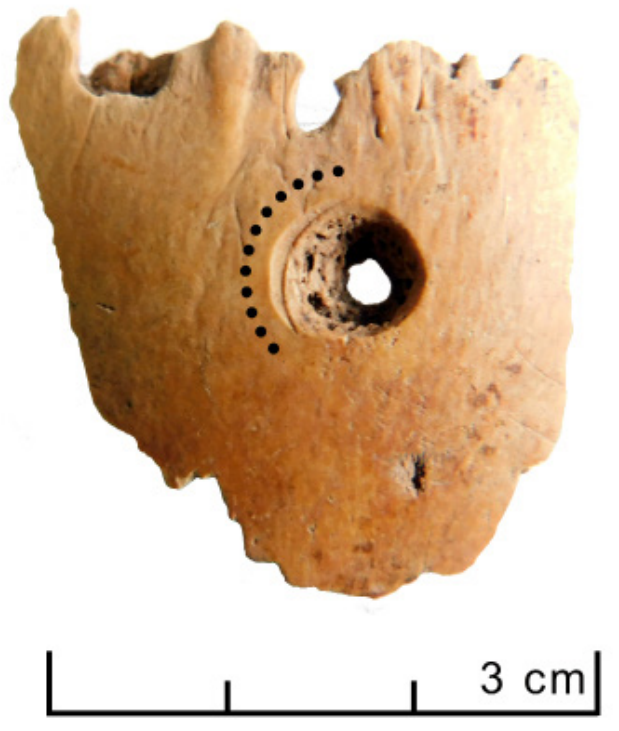

Figura 5. Detalle del escalón registrado en el borde de la oquedad correspondiente al fragmento $\mathrm{B}$.

Detail of the step registered at the edge of the cavity (fragment B).

ausencia de cicatrización, corroborada macroscópica y radiológicamente, posibilitó determinar de manera clara la técnica empleada. Las características de las oquedades, es decir sus formas circulares, secciones tronco-cónicas y diámetros pequeños, claramente indican que la intervención fue realizada a partir de la técnica de perforación. Según nuestros análisis, las cinco oquedades del parietal derecho habrían sido efectuadas con un instrumento punzante y probablemente a través de un movimiento giratorio, tal como se desprende de la huella de manufactura registrada en una de las perforaciones. Resulta relevante mencionar la estandarización de las cinco oquedades observadas y el hecho de tratarse de un hueso muy fino por pertenecer a un subadulto. A partir de estos aspectos, entonces, es sugerente pensar en la gran habilidad y el conocimiento de la técnica que poseía la persona que las llevó a cabo; este punto se retomará más adelante. Como se mencionó previamente, entre los antecedentes publicados que describen esta práctica en otros grupos de Argentina, existen ejemplos reportados para Patagonia y para el Noroeste (Castro et al. 2009; Fuchs et al. 2017). En ambos casos las perforaciones presentes en el cráneo fueron interpretadas como trepanaciones, las cuales se efectuaron con propósitos terapéuticos. En el ejemplo de Patagonia, al igual que el presentado en este trabajo, la técnica elegida para realizar la intervención fue la perforación.

Varios autores hacen referencia a que existe una relación entre el tipo de técnica empleada para hacer las oquedades y la cicatrización de las mismas (Campillo 2011; Giuffra y Fornaciari 2017). Como señalan Erdal y Erdal (2011), la mayoría de los casos con ausencia de cicatrización son aquellos en los que la técnica usada fue la perforación. Tales intervenciones pudieron haber ocurrido tanto en momentos premortem, con poca sobrevida del individuo (sin dar lugar a la respuesta ósea), como en momentos posteriores e inmediatos a la muerte. Las trepanaciones realizadas por raspado o corte, en tanto, han mostrado mayor número de individuos con remodelación ósea en los márgenes de las aberturas, indicando la sobrevida de estos luego de las intervenciones. En el caso presentado en este trabajo, las celdas del diploe visibles y la ausencia de actividad osteogénica serían coincidentes con la relación entre la técnica empleada de perforación y la no cicatrización. Por otro lado, la ausencia de grietas y minifracturas en los bordes de las oquedades indica que el hueso se encontraba en estado fresco en el momento de realizar las perforaciones. En este sentido, no es posible determinar si tales invervenciones fueron realizadas premortem o inmediatamente después de la muerte.

Luego de haber identificado como antrópicas las oquedades registradas en fragmentos craneanos del infante CL2-80, que estas fueron efectuadas mediante la técnica de perforación y que la intervención se habría llevado a cabo en momentos perimortem, uno de los temas más complejos de analizar se relaciona con los motivos que condujeron a realizar esta práctica. Como fue referido anteriormente, en el caso de las trepanaciones terapéuticas, la determinación es más simple, sobre todo cuando se cuenta con el esqueleto completo y se pueden verificar los traumas relacionados o hay lesiones patológicas presentes (Gresky et al. 2016). En contraposición, cuando las intervenciones se realizan con fines no médicos, como rituales o mágicos, no suele haber evidencia material claramente visible en el esqueleto (Gresky et al. 2016; Slepchenko et al. 2017). Para el caso del infante presentado en este trabajo, ambas motivaciones son abordadas a continuación con respecto a las evidencias encontradas. En cuanto a la explicación basada en fines terapéuticos, en este individuo no se observaron evidencias de lesiones traumáticas o patológicas que pudieran ser las causas de las perforaciones. Sin embargo, hay que considerar que 
el esqueleto está conformado en este caso solo por fragmentos del cráneo y la mandíbula, por lo que es posible que esta subrepresentación actúe sesgando el relevamiento patológico y no pueda descartarse completamente el fin terapéutico. En relación con los motivos rituales, tal como fue apuntado por varios autores, la presencia de perforaciones sin cicatrización y vinculadas con su realización en momentos postmortem indica fuertemente el carácter ritual de este tipo de intervención (Buzhilova et al. 2006; Campillo 1984, 2011; Giuffra y Fornaciari 2017; Gresky et al. 2016; Lisowski 1967). Entre los motivos rituales, lo más destacado en la bibliografía es el uso de piezas del cráneo con el propósito de utilizarlas como amuletos. Se ha sugerido que las piezas de hueso extraídas durante estas operaciones tenían un poderoso significado mágico y ceremonial (Erdal y Erdal 2011; Khudaverdyan 2014). Distintos ejemplos de Armenia y Rusia meridional aluden a que los amuletos craneales producidos con fragmentos de cráneo humano fueron usados o exhibidos como colgantes, posiblemente para atraer fuerza, dar protección o como una consideración especial hacia el difunto (Gresky et al. 2016; Khudaverdyan 2014, 2016). En el infante analizado en este trabajo, si bien no puede confirmarse con tal certeza que la intervención se haya efectuado postmortem, la presencia de varios orificios sin cicatrización y la ausencia de patologías asociadas permiten plantear el posible carácter ritual de la intervención como la explicación más plausible. En cuanto a la confección de amuletos craneales, es sugerente que los fragmentos de cráneo perforado del infante CL2-80 se encontraran junto al cráneo del individuo adulto $\mathrm{n}^{\circ} 78$.

$\mathrm{Si}$ se considera la posibilidad de que los fragmentos del cráneo perforado de CL2-80 fueron utilizados como un amuleto y enterrados junto a un adulto masculino, es necesario discutir las características de los acompañamientos mortuorios de los otros sitios del área vinculados con la entidad arqueológica GoyaMalabrigo, con la cual fue identificada la ocupación en el sitio CL2. Los trabajos bioarqueológicos llevados a cabo en los sitios asignados a la entidad hacen referencia a la asociación de los entierros con objetos de diversa ergología. Entre los diferentes ejemplos se puede mencionar el acompañamiento de los entierros con restos faunísticos (cráneos y colmillos de félidos), materiales cerámicos (contenedores, vasos), instrumentos líticos y óseos, cuentas de collar, entre otros (ver síntesis en Ramos van Raap 2018b).
Una mención especial merece el sitio La Palmera II, localizado en el Paraná Medio (Provincia de Santa $\mathrm{Fe}$, Argentina), donde se halló un paquete funerario asociado a distintos objetos (instrumentos en hueso, cuentas de collar, apéndices cerámicos, una plaqueta de cobre perforada) y a elementos óseos humanos (Ceruti 2018). Específicamente, se encontró junto al entierro secundario una mano articulada, dientes y falanges humanas, que fueron interpretados como posibles sacrificios humanos (Ceruti 1993). Este ejemplo constituye hasta el momento el único antecedente de un sitio de la entidad Goya-Malabrigo donde se registró el uso de restos humanos como acompañamiento de los entierros. En este sentido, el caso dado a conocer en este artículo ampliaría tal registro para esta entidad arqueológica. Por otro lado, si consideramos que las oquedades registradas en CL2-80 se realizaron en momentos inmediatos a la muerte, estas acciones indicarían una importante manipulación de los cuerpos luego del deceso. En este punto es necesario mencionar que una de las características recurrentes en los sitios asignados a Goya-Malabrigo es la alta manipulación de los entierros, que se refleja en la selección de partes esqueletarias, desarticulación de los esqueletos, eliminación de tejido blando, formación de paquetes secundarios, relocalización de elementos óseos y aplicación de ocre (Ramos van Raap 2018b; Scabuzzo y Ramos van Raap 2017).

Finalmente, como ya se mencionó, en América del Sur la mayor cantidad de casos de cráneos perforados se vinculan con la realización de trepanaciones y fueron registrados en las tierras altas, particularmente en la frontera entre Perú y Bolivia (Verano 1997). Cabría entonces discutir el origen geográfico del individuo analizado aquí. Por una parte, es posible plantear que la técnica de perforación pudo ser desarrollada de manera local. Por otra cabe la posibilidad de que quien practicó tal intervención o que el mismo infante inhumado en CL2 proceda de aquellas regiones donde este tipo de prácticas de perforación de los cráneos se realizaban. En cuanto a la primera posibilidad, en el registro arqueológico del área sur del Noreste Argentino se registra la presencia de perforaciones en artefactos óseos faunísticos, en dientes de cánidos, en valvas de moluscos y en cerámica. Estas perforaciones son, principalmente, de formas circulares e incluso algunas con diámetro externo mayor al interno (Acosta et al. 2015; Bonomo et al. 2009; Castro 2017; Ceruti 2003; Di Prado 2015; Politis et al. 2017, entre otros). Por consiguiente, se puede suponer que, aunque no 
hay otros registros de la aplicación de la técnica en restos humanos, existía un conocimiento a nivel local de cómo realizar las perforaciones. Este aspecto se relaciona con lo anteriormente señalado relativo a la estandarización de las formas y tamaños de las oquedades aquí analizadas y la habilidad de quien las efectuó. En cuanto a la segunda posibilidad, esto es, su origen, queda planteada la duda de si los fragmentos de cráneo perforado proceden de un área distinta de aquella donde esta práctica se encontraba desarrollada, como es el caso de las tierras altas sudamericanas. En este sentido es conocida una red de circulación de bienes, personas y conocimientos que conectaba el Noreste de Argentina con otras regiones de Sudamérica (ver Bonomo et al. 2017). Para echar luz sobre este punto los futuros análisis bioarqueológicos debieran orientarse al análisis del ADN del individuo con el fin de conocer su procedencia.

\section{Conclusiones}

En este trabajo se presentó el primer caso de perforaciones intencionales en el cráneo de un individuo infantil inhumado en un sitio del Noreste Argentino, al mismo tiempo que constituye uno de los pocos registros prehispánicos de este tipo de intervención conocido para Argentina. En la evaluación de estas oquedades resultó crucial la realización de radiografías y del diagnóstico diferencial, en el que se consideraron distintas patologías óseas, traumas y efectos postdepositacionales. El análisis efectuado también permitió inferir el tipo de técnica empleada para la intervención; la regularidad en la morfología de las oquedades y la dificultad de ejecución, tratándose de un soporte frágil y delicado, posibilitan suponer que la persona encargada de realizarla poseía habilidades desarrolladas para esta tarea.
Este caso de estudio es interpretado como resultado de la práctica intencional de modificación del cráneo, probablemente efectuada en momentos inmediatamente posteriores a la muerte del individuo y con un probable fin ritual vinculado a la confección de amuletos. Aun así, no puede descartarse completamente la intervención premortem $\mathrm{y}$, sin sobrevida, del infante. La principal limitación en la interpretación de este caso radica en que se trata de un único ejemplar y que no hay evidencias de perforaciones intencionales del cráneo en otros sitios de la región. Asimismo, en la bibliografía sobre el tema, es poco frecuente la presencia de estas prácticas en individuos de corta edad, lo que constituye la singularidad del caso de estudio aquí expuesto. Por otro lado, de tratarse de una práctica con fines rituales, las perforaciones registradas representan uno de los pocos ejemplos de este tipo de práctica conocida en Sudamérica, donde generalmente las oquedades intencionales del cráneo corresponden a la realización de trepanaciones efectuadas en individuos vivos y con fines terapéuticos. Esperamos que los futuros análisis bioarqueológicos permitan descubrir otros casos semejantes al presentado para, de esta manera, poder ahondar en estos análisis.

Agradecimientos: Las autoras desean agradecer al Dr. John Verano por su generosidad en la consulta sobre el caso de estudio y en brindar bibliografía. A la Dras. Paula Campos y Roxana Mariani por la consulta entomológica realizada. A la Dra. Rocío García Mancuso por su ayuda para la identificación de los fragmentos craneales. $\mathrm{Al}$ equipo de radiología del Instituto Médico Privado de Diamante por facilitar la realización de las radiografías. Al Dr. Rodrigo Angrizani por su colaboración con las imágenes. Finalmente, a los tres evaluadores anónimos por sus comentarios y sugerencias que ayudaron a mejorar el manuscrito.

\section{Referencias Citadas}

Acosta, A., N. Buc, M. Ramírez, F. Prevosti y D. Loponte 2015. Producción y uso de objetos ornamentales elaborados sobre dientes de carnívoros en contextos arqueológicos del Humedal de Paraná Inferior. Revista del Museo de Antropología 8 (2):33-46.

Andrushko, V. y J. Verano 2008. Prehistoric Trepanation in the Cuzco Region of Peru: A View into an Ancient Andean Practice. American Journal of Physical Anthropology 137: 4-13.

Aufderheide, A.C. y C. Rodriguez-Martin 1998. The Cambridge encyclopedia of human paleopathology. Cambridge University Press, Cambridge.

Bonomo, M., E.D. Cabanillas y R. Montero 2017. Archaeometallurgy in the Paraná Delta (Argentina): Composition, manufacture, and indigenous routes. Journal of Anthropological Archaeology 47:1-11

Bonomo, M., I. Capdepont y A. Matarrese 2009. Alcances en el estudio de colecciones. Los materiales arqueológicos del Delta del río Paraná depositados en el Museo de La Plata (Argentina). Arqueología Sudamericana 5:68-101.

Bonomo, M., V. Di Prado, C. Silva, C. Scabuzzo, M.A. Ramos van Raap, C. Castiñeira y G.G. Politis 2019. Las poblaciones indígenas prehispánicas del Paraná Inferior y Medio. Revista del Museo de La Plata 4 (2):585-620.

Buikstra J. y D. Ubelaker 1994. Standards for Data Collection from Human Skeletal Remains. Arkansas Archeological Survey, Arkansas. 
Buzhilova, A.P., M.V. Dobrovolskaya y M. B. Mednikova 2006. On the problem of the reconstruction of the social relations of the population of the Baraba Steppe (analysis of injury or damage for the anthropological material Series Sopka-2). Archaeology, Ethnology and Anthropology of Eurasia 3:148-156.

Campillo, D. 1984. Neurosurgical pathology in prehistory. Acta Neurochirurgica 70:275-290.

Campillo, D. 2007. La Trepanación Prehistórica. Ediciones Bellaterra, Barcelona.

Campillo, D. 2011. La trepanación prehistórica en la Península Ibérica. En Paleopatología: Ciencia Multidisciplinar, editado por A. González Martín, pp. 1-24. Sociedad Española de Paleopatología, Madrid.

Castro, J.C. 2017. Investigaciones Arqueológicas en la Cuenca Media e Inferior del Río Uruguay. Tesis doctoral inédita, División Arqueología, Facultad de Ciencias Naturales y Museo, Universidad Nacional de La Plata, La Plata.

Castro, J.C. y M. Del Papa 2015. La estructura del registro bioarqueológico del río Uruguay inferior. Análisis de la colección osteológica humana del Museo Arqueológico Manuel Almeida (Gualeguaychú, Entre Ríos). Intersecciones en Antropología 16:195-205.

Castro, A., S. Salceda, M. Plischuk y B. Desántolo 2009. Bioarqueología de rescate: Sitio Carsa (Costa Norte de Santa Cruz, Argentina). En Arqueología de Patagonia: Una Mirada Desde el Último Confín, compilado por M. Salemme, F. Santiago, M. Álvarez, E. Piana, M. Vázquez y E. Mansur, Tomo II, pp. 629-638. Editorial Utopías, Ushuaia.

Ceruti, C.N. 1993. Arqueología. En Nueva Enciclopedia de la Provincia de Santa Fe, editado por A. Renna, pp. 557-580. Sudamérica, Santa Fe.

Ceruti, C.N. 2003. Entidades culturales presentes en la cuenca del Paraná Medio (margen entrerriana). Mundo de Antes 3:111-135.

Ceruti, C.N. 2018. Indicios de complejidad social en la entidad arqueológica Goya-Malabrigo: los enterratorios humanos del sitio La Palmera II (Hernandarias, departamento Paraná, provincia de Entre Ríos, Argentina). En Goya Malabrigo: Arqueología de una Sociedad Indígena del Noreste Argentino, editado por G. Politis y M. Bonomo, pp. 149-173. Editorial UNICEN, Tandil.

Comşa, A. y A. Sankhyan 2013. Amazing skills: Practice of trepanation around the world. En Recent Discoveries and Perspectives in Human Evolution, editado por A. Sankhyan, pp. 173-182. Oxford Archaeopress, Oxford.

Costa Angrizani, R. y C. Scabuzzo 2019. Múltiples enfoques para abordar la alimentación en los contextos de ocupación guaraní del Río Uruguay inferior. Trabajo presentado en el VIII Encuentro de Discusión de Arqueología del Nordeste Argentino, Posadas, Octubre 2019.

Di Prado, V. 2015. Estudio Comparativo de las Prácticas de Elaboración y Uso de la Alfarería Prehispánica del Centro-Este de Argentina desde una Perspectiva Macrorregional. Tesis doctoral inédita, División Arqueología, Facultad de Ciencias Naturales y Museo, Universidad Nacional de La Plata, La Plata.

Erdal, Y.S. y Ö.D. Erdal 2011. A review of trepanations in Anatolia with new cases. International Journal of Osteoarchaeology 21:505-534.
Fuchs, M.L., P. Miranda De Zela y S. Rodríguez-Curletto 2017. Doncellas: estudio bioarqueológico de una colección osteológica de la Puna de Jujuy, Argentina. Actas de la VII Reunión de la Asociación de Paleopatología en Sudamérica. Vida y Muerte en el Desierto de Atacama, pp. 246-247. Asociación de Paleopatología Sudamericana, Arica.

Giuffra, V. y G. Fornaciari 2017. Trepanation in Italy: A review. International Journal of Osteoarchaeology 27:745-767.

Gresky, J., E. Batieva, A. Kitova, A. Kalmykov, A. Belinskiy, S. Reinhold y N. Berezina 2016. New cases of trepanations from the 5th to 3rd millennia BC in Southern Russia in the context of previous research: Possible evidence for a ritually motivated tradition of cranial surgery? American Journal of Physical Anthropology 160 (4):665-682.

Huchet, J.B., F. Le Mort, R. Rabinovich, S. Blau, H. Coqueugniot y B. Arensburg 2013. Identification of dermestid pupal chambers on Southern Levant human bones: Inference for reconstruction of Middle Bronze Age mortuary practices. Journal of Archaeological Science 40:3793-3803.

Khudaverdyan, A. 2014. Post-mortem trepanations in a burial in Late Bronze Age and Iron Age from Armenian plateau. Antropologija 14 (3):75-91.

Khudaverdyan, A. 2016. Trepanation in the Late Bronze Age and Early Iron Age in Armenia. HOMO - Journal of Comparative Human Biology 67:447-461.

Kurin, D. 2013. Trepanation in South-Central Peru during the Early Late Intermediate period (ca. AD 1000-1250). American Journal of Physical Anthropology 152:484-494.

Lisowski, F.P. 1967. Prehistoric and early historic trepanation. En Diseases in Antiquity, editado por D. Brothwell y A.T Sandison, pp. 651-672. C.C. Thomas, Springfield.

Luis, M. y H. Pucciarelli 1996. Trepanación craneana: Una práctica frecuente en América precolombina. Revista Museo 7:71-76.

Mariani-Costantini, R., P. Catalano, F. di Gennaro, G. di Tota y L.R. Angeletti 2000. New light on cranial surgery in ancient Rome. The Lancet 355:305-307.

Nicklisch, N., V. Dresely, J. Orschiedt, F. Ramsthaler, B. Schlenker, R. Ganslmeier, S. Friederich, H. Meller y K.W. Alt 2018. A possible case of symbolic trepanation in Neolithic Central Germany. International Journal of Osteoarchaeology 28 (3):216-226.

Ortner, D.J. 2003. Identification of Pathological Conditions in Human Skeletal Remains. Academic Press, Amsterdam.

Piek, J., G. Lidke, T. Terberger, U. von Smekal y M.R. Gaab 1999. Stone age skull surgery in Mecklenburg-Vorpommern: a systematic study. Neurosurgery 45:147-151.

Podgorny, I. 2016. Trepanaciones Peruanas. Fundación Espigas, Buenos Aires.

Politis, G. y M. Bonomo 2012. La entidad arqueológica GoyaMalabrigo (Ríos Paraná y Uruguay) y su filiación Arawak. Boletín de la Sociedade de Arqueologia Brasileira (SAB) 25 (1):10-46.

Politis, G. y M. Bonomo (eds.) 2018. Goya-Malabrigo: Arqueología de una Sociedad Indígena del Noreste Argentino. Editorial UNICEN, Tandil. 
Politis, G., L. Bastourre, V. Di Prado, M. Bonomo, G. Moreira y A. Matarrese 2017. El Túmulo II del Brazo Largo. Aportes para la arqueología del Delta Inferior del río Paraná. Revista del Museo de Antropología 10 (2):71-88.

Ramos van Raap, M.A. 2018a. Paleopatología en Poblaciones Prehispánicas del Sur del Noreste Argentino. Tesis doctoral inédita, División Arqueología, Facultad de Ciencias Naturales y Museo, Universidad Nacional de La Plata, La Plata.

Ramos van Raap, M.A. 2018b. El registro mortuorio de la entidad arqueológica Goya-Malabrigo. En Goya-Malabrigo: Arqueología de una sociedad Indígena del Noreste Argentino, editado por G. Politis y M. Bonomo, pp. 175-199. Editorial UNICEN, Tandil.

Ramos van Raap, M.A. y C. Scabuzzo 2018. Estudios bioarqueológicos de la colección del Museo Arqueológico Manuel Almeida (provincia de Entre Ríos). Relaciones de la Sociedad Argentina de Antropología XLIII (2):185-205.

Riccomi, G., G. Fornaciari, A. Vitiello, A. Bini, D. Caramella y V. Giuffra 2017. Trepanation to Treat a Head Wound: A Case of Neurosurgery from 13th-Century Tuscany. World Neurosurgery 104:9-13.

Scabuzzo, C. y M.A. Ramos van Raap 2017. Nuevos resultados de los estudios osteológicos del sitio Los Tres Cerros 1 (Delta Superior del río Paraná). Comechingonia. Revista de Arqueología 21 (2):201-228.

Scabuzzo, C. y M.A. Ramos van Raap 2019. Alcances y limitaciones en el relevamiento de osteoartrosis. Primeros resultados de los análisis en colecciones osteológicas del sur del Noreste Argentino. En Metodologías para el Estudio de Restos Esqueletales en Argentina: Actualizaciones y Nuevas Perspectivas, editado por M. Fabra, P. Novellino, M. Arrieta y S. Salega, pp. 97-124. Facultad de Filosofía y Humanidades, UNC, Córdoba.
Slepchenko, S., A.V. Vybornov, V.S. Slavinsky, A.A. Tsybankov y V.E. Matveev 2017. Ante mortem cranial trepanation in the Late Bronze Age in Western Siberia. International Journal of Osteoarchaeology 27:356-364.

Ubelaker, D. 1987. Estimating age at death from immature human skeletons: An overview. Journal of Forensic Sciences $32: 1254-1263$

Valenzuela Jiménez, G. y M.A. Santos Ramírez 2013. Cráneos perforados de dos sitios prehispánicos de la frontera mesoamericana. Cerro del Huistle, Jalisco, y La Quemada, Zacatecas. Un análisis comparativo. Estudios de Antropología Biológica XVI:207-232.

Velasco-Suarez, M., J.B. Martinez, R. García Oliveros y P.R. Weinstein 1992. Archaeological origins of cranial surgery: Trephination in Mexico. Neurosurgery 31:313-319.

Verano, J. 1997. La trepanación como tratamiento terapéutico para fracturas craneales en el antiguo Perú. Estudios de Antropología Biológica 8:65-81.

Verano, J. 2016a. Holes in the Head. The Art and Archaeology of Trepanation in Ancient Peru. Dumbarton Oaks Research Library and Collection, Washington, D.C.

Verano, J. 2016b. Differential diagnosis: Trepanation. International Journal of Paleopathology 14:1-9.

Weber, J. y J Whal 2006. Neurosurgical Aspects of Trepanations from Neolithic Times. International Journal of Osteoarchaeology 16:536-545.

White, T.D. y P.A. Folkens 2005. The Human Bone Manual. Elsevier Academic Press, Amsterdan, Boston. 
